\title{
Review of: "Online Reference Trajectory Adaptation: A Personalized Control Strategy for Lower Limb Exoskeletons"
}

\author{
Hubert Gattringer ${ }^{1}$ \\ 1 Johannes Kepler University Linz
}

Potential competing interests: The author(s) declared that no potential competing interests exist.

The authors present an approach for the adaptation of the trajectory of a lower body exoskeleton to the gait motion of the human user. Thereby, the adaption rule is based on a cost function that penalizes the interaction force between human and robot, as well as the deviation between a reference trajectory and the human intended motion. The presented method showed stable behavior with simulated healthy and motor-impaired subjects, while increasing gait stability and improving different gait parameters.

The methods are clearly formulated and the results are discussed adequately. While I see no major flaws in the scientific basis of this work, I do have a minor remark to the authors:

- Page 2, Equations (4) and (5): I believe the factor 1/2 in (5) should not appear in this equation as it already occurs in (4), which is sufficient to cancel out when the cost function $\mathrm{J}$ is differentiated 